\title{
Nível de Atividade Física e Estado de Humor em Adolescentes
}

\author{
Francisco Zacaron Werneck ${ }^{1}$ \\ Programa de Pós-Graduação em Métodos Estatísticos Computacionais - UFJF \\ Cristiane Amorim Navarro \\ Prefeitura Municipal de Santana do Deserto
}

\begin{abstract}
RESUMO - Poucos estudos investigaram a relação exercício-humor em adolescentes. O objetivo do presente estudo foi verificar a relação entre o nível de atividade física relatado por adolescentes e o estado de humor, analisando diferenças entre gêneros. Dezessete meninos $(\mathrm{M}=17,5 ; \mathrm{DP}=1,4$ anos) e 24 meninas $(\mathrm{M}=16,0 ; \mathrm{DP}=2$ anos) foram avaliados na escola. Mensurou-se o nível de atividade física habitual (NAFH) e o estado de humor. Verificou-se relação negativa entre o NAFH e o distúrbio total de humor $(r=-0,30, p<0,05)$, bem como relação positiva entre o NAFH e Vigor $(r=0,48, p<0,01)$, tanto nas meninas quanto nos meninos. Conclui-se que os participantes que relataram maior nível de atividade física apresentaram melhor escore no estado de humor, independente do gênero.
\end{abstract}

Palavras-Chave: exercício; atividade física; humor; saúde psicológica.

\section{Physical Activity Level and State of Mood in Adolescents}

\begin{abstract}
A relative small number of studies about the relationship between state of mood and exercise in adolescents have been published. The aim of the present study was to verify the relationship between informed physical activity by adolescents and the state of mood. Seventeen boys ( $\mathrm{M}=17,5 ; \mathrm{DP}=1,4$ years) and 24 girls $(\mathrm{M}=16,0 ; \mathrm{DP}=2,0$ years) were evaluated at school. The habitual physical activity level (HFAL) and mood states were measured. The analyses showed a negative correlation between HFAL and total mood disturbance $(r=-0,30, p<0,05)$ as well as a positive correlation between HFAL and vigour $(r$ $=0,48, \mathrm{p}<0,01)$ in boys and girls. In conclusion, adolescents with related high level of physical activity showed better mood state, independent of gender.
\end{abstract}

Keywords: exercise; physical activity; mood; mental health

Tem sido demonstrado que a prática regular da atividade física está associada a diversos benefícios psicológicos, como menor reatividade ao estresse, diminuição da ansiedade, depressão e hostilidade, melhoria do humor, do autoconceito e do bem-estar psicológico, tanto em pessoas saudáveis quanto em pacientes clínicos (Dunn, Trivedi \& O’Neal, 2001; Petruzzello, Landers, Hatfield, Kubitz \& Salazar, 1991; U.S.Department of Health and Human Services, 1996). De todas as técnicas comportamentais usadas para a regulação do humor, o exercício mostrou-se a mais efetiva na alteração de um mau humor, a quarta mais bem sucedida no aumento do vigor e a terceira na redução da tensão (Thayer, Newman \& McClain, 1994). Em face de todas essas evidências, o exercício físico tem se tornado uma alternativa não-farmacológica, econômica, saudável e de maior adesão na prevenção e tratamento de distúrbios psicológicos e na promoção da saúde mental.

Os benefícios psicológicos gerados pela prática do exercício encontram-se muito difundidos por profissionais da área da saúde, meios de comunicação e pesquisadores, denotando a importância social e acadêmica do tema. $\mathrm{O}$ crescente número de estudos sobre os efeitos psicológicos do exercício tem se dado, por um lado, na medida em que a saúde deixou de ser considerada uma condição meramente física e passou a ser concebida como uma interação de aspectos físicos,

1 Endereço para correspondência: Francisco Zacaron Werneck R: Euclides Dantas Werneck, 28 - Centro Comendador Levy Gasparian - RJ. CEP 25870-000 E-mail: fzacaron@oi.com.br psicológicos e sociais, na qual a saúde mental é crucial para o bem-estar geral dos indivíduos; e, por outro lado, a partir do momento em que os estudos na área de atividade física passaram a considerar estas interações, revelando implicações em diferentes cenários.

Uma das variáveis psicológicas mais estudadas na área do exercício, a partir da década de 1970, foi o estado de humor, após o desenvolvimento e a validação do questionário Perfil dos Estados de Humor - POMS (McNair, Lorr \& Droppleman, 1971). Embora ainda não exista uma definição consensual, o estado de humor pode ser entendido como um estado psicológico composto por sentimentos positivos e negativos, mensurados pelo POMS, que variam em intensidade e duração, sendo um indicador do bem-estar psicológico (Werneck, 2003).

Em geral, pessoas fisicamente ativas e com maior aptidão física possuem um melhor estado de humor do que aquelas sedentárias e menos aptas. Estudos transversais que compararam variáveis psicológicas de indivíduos ativos e sedentários e entre aqueles com diferentes níveis de atividade e aptidão física corroboram essa premissa (Hassmén, Koivula \& Uutela, 2000; Hills \& Argyle, 1998; Lobstein, Mosbacher \& Ismail, 1983; Stephens, 1988; Thirlaway \& Benton, 1992). Além disso, baixos níveis de atividade física predisseram sintomas depressivos, tendo o exercício um efeito protetor contra a incidência desses sintomas em idades superiores (Lampinen, Heikkinen \& Ruoppila, 2000; Strawbridge, Deleger, Roberts \& Kaplan, 2002). 
Podem-se destacar duas hipóteses para a relação exercício e humor: 1) pessoas ativas podem reportar melhor saúde mental porque a atividade física melhora a saúde mental; 2) pessoas com melhor saúde mental tendem a ser mais ativas. Inúmeros estudos experimentais disponíveis suportam a primeira hipótese, revelando que o exercício é um poderoso promotor de saúde mental (DiLorenzo et al., 1999; Hansen, Stevens \& Coast, 2001; Steinberg et al., 1998; Werneck, 2003).

Apesar da associação positiva entre exercício e saúde psicológica, algumas variáveis, como o nível de atividade física, a idade e o gênero dos praticantes, podem moderar o grau dessa relação. Pelas evidências disponíveis, observam-se poucos estudos que analisaram a relação entre o nível de atividade física e o estado de humor em jovens adolescentes, havendo controvérsias quanto à diferença de humor entre os gêneros (Werneck, 2003). Quanto a este aspecto, os estudos de McNair et al. (1971), McAuley e Rudolph (1995) e Terry e Lane (2000) não verificaram diferenças no estado de humor em função do exercício em homens e mulheres ou em indivíduos de diferentes idades, ao contrário dos achados de Terry, Lane e Keohane (1999). Além da questão gênero, ainda não se determinou um efeito de dose-resposta entre exercício e saúde psicológica. Não se sabe até que ponto uma maior quantidade de atividade física é mais benéfica do que uma menor quantidade.

Neste sentido, o objetivo do presente estudo foi verificar a relação entre o nível de atividade física relatado por adolescentes e o escore obtido em um teste que avalia o estado de humor (POMS), analisando possíveis diferenças entre os gêneros.

\section{Método}

\section{Participantes}

Participaram do estudo ( $\mathrm{M}=17,5 ; \mathrm{DP}=1,4$ anos $)$ e 24 meninas $(M=16,0 ; D P=2$ anos), estudantes do ensino fundamental e médio, de uma escola estadual do município de Três Rios - RJ, que participaram das avaliações do estande de Educação Física durante uma feira de ciências, realizada na escola.

\section{Instrumentos}

As seguintes variáveis foram mensuradas: peso, altura, percentual de gordura, pressão arterial, freqüência cardíaca de repouso, nível de atividade física habitual e estado de humor. Para a mensuração da variável peso utilizou-se uma balança mecânica com precisão de 100g. Para altura, usou-se uma fita métrica fixada à parede. $\mathrm{O}$ percentual de gordura corporal foi estimado a partir das dobras cutâneas tríceps e panturrilha, através da equação de Slaughter et al. (1988). A pressão arterial foi mensurada pelo método auscultatório. A freqüência cardíaca foi mensurada a partir de um monitor de freqüência cardíaca Polar Vantageâ. O nível de atividade física habitual (NAFH) foi estimado a partir do Questionário de Atividades Físicas Habituais (Nahas, 2001) que, segundo este autor, mostrou-se válido e fidedigno entre adolescentes e universitários. O questionário é composto por questões sobre atividades ocupacionais diárias e atividades de lazer. Para cada resposta positiva existe uma pontuação que ao final é somada, sendo fornecido um valor indicativo de quanto ativo é o indivíduo. A pontuação final varia de zero a 21 ou mais pontos, sendo 12 a 20 pontos o escore correspondente a uma pessoa fisicamente ativa.

O estado de humor foi mensurado pelo POMS - Perfil dos Estados de Humor (Brandão, Andrade, Matarazo, Vasques, \& Vasconcelos, 1993). Terry e Lane (2000) reportaram valores de consistência interna do POMS que variaram entre 0,72 a 0,84 . O POMS avalia seis estados subjetivos de humor: tensão $(T)$, depressão $(\mathrm{D})$, raiva $(\mathrm{R})$, vigor $(\mathrm{V})$, fadiga $(\mathrm{F}) \mathrm{e}$ confusão mental (C). Os fatores T, D, R, F e C são considerados os fatores negativos do humor, sendo o vigor considerado o fator positivo. O distúrbio total de humor (DTH) é calculado pela soma dos fatores negativos, menos o fator positivo (DTH $=(\mathrm{T}+\mathrm{D}+\mathrm{R}+\mathrm{F}+\mathrm{C})-\mathrm{V})$, sendo uma medida global do distúrbio de humor. Ao resultado final do DTH, foi somado 100 para que não houvessem resultados negativos. O perfil de humor com alto valor de vigor e baixos valores para as outras variáveis é denominado "perfil iceberg", sendo representativo de uma saúde mental positiva. O POMS é um questionário de auto-relato composto por 65 adjetivos em uma escala tipo Likert de 5 pontos, onde $0=$ nada e $4=$ extremamente. Foi orientado aos participantes responderem de acordo como eles estavam se sentindo "agora, neste exato momento".

\section{Procedimentos}

Todos os alunos participantes da feira de ciências estavam cientes da realização das medidas e avaliações que seriam realizadas no estande de educação física. Os interessados em participar dos testes foram instruídos sobre os procedimentos e sobre o objetivo do estudo, que era analisar a relação entre a prática habitual de exercício e o estado de humor em adolescentes. Inicialmente, eles preencheram uma ficha contendo dados pessoais, subseqüentemente respondendo ao POMS, ao Questionário de Atividades Físicas Habituais, à medida da freqüência cardíaca de repouso, da pressão arterial, do peso, da altura e do percentual de gordura corporal. O estudo foi aprovado pelo Comitê de Ética da Universidade Federal de Juiz de Fora.

\section{Análise Estatística}

Inicialmente, foi utilizada uma análise descritiva dos dados (Média e Desvio-Padrão). Posteriormente, usou-se a correlação de Pearson para as variáveis nível de atividade física habitual e estado de humor, considerando $\mathrm{p}<0,05$, como nível de significância para a correlação. Um teste t para medidas independentes foi usado para comparar diferenças entre gênero para as variáveis estudadas.

\section{Resultados}

Os dados descritivos (média e desvio-padrão) referentes às características dos sujeitos e às variáveis estudadas podem ser vistos na Tabela 1 . 
Tabela 1: Características morfofuncionais de estudantes do ensino fundamental e médio de uma escola estadual da cidade de Três Rios - RJ.

\begin{tabular}{lll}
\hline Variável & $\begin{array}{l}\text { Meninos } \\
(\mathrm{n}=17)\end{array}$ & $\begin{array}{l}\text { Meninas } \\
(\mathrm{n}=24)\end{array}$ \\
\hline Idade (anos) & $17,5 \pm 1,4$ & $16 \pm 2,0^{* *}$ \\
\hline Peso $(\mathrm{Kg})$ & $67,1 \pm 5,9$ & $51,3 \pm 8,4^{* *}$ \\
\hline Altura $(\mathrm{m})$ & $1,77 \pm 0,2$ & $1,61 \pm 0,15^{* *}$ \\
\hline IMC $\left(\mathrm{Kg} / \mathrm{m}^{2}\right)$ & $21,3 \pm 1,6$ & $19,6 \pm 2,4^{* *}$ \\
\hline \%Gordura Corporal & $8,7 \pm 4,5$ & $19,1 \pm 4,9^{* *}$ \\
\hline Freqüência Cardíaca (bpm) & $75,7 \pm 9,8$ & $77,9 \pm 6,3$ \\
\hline $\begin{array}{l}\text { Pressão Arterial Sistólica } \\
\text { (mmHg) }\end{array}$ & $122,3 \pm 13$ & $105,7 \pm 9,8^{* *}$ \\
\hline $\begin{array}{l}\text { Pressão Arterial Diastólica } \\
\text { (mmHg) }\end{array}$ & $80,6 \pm 13,4$ & $68,8 \pm 8,0^{* *}$ \\
\hline $\begin{array}{l}\text { Nível de Atividade Física } \\
\text { Habitual }\end{array}$ & $17,5 \pm 8,7$ & $12 \pm 7,6^{*}$ \\
\hline
\end{tabular}

$(*$ : diferente para $\mathrm{p}<0,05 ; * *$ : diferente para $\mathrm{p}<0,01)$
Em média, o grupo de alunos estudados, de acordo com o questionário adotado, pode ser considerado ativo. O teste $\mathrm{t}$ de Student para medidas independentes revelou que os meninos apresentam maior nível de atividade física habitual do que as meninas $(\mathrm{p}<0,05)$; entretanto, não foram encontradas diferenças estatisticamente significativas entre o perfil do estado de humor de meninos e meninas, embora os meninos tenham apresentado uma tendência de melhor perfil de humor.

Foi observada uma relação positiva entre o vigor e o nível de atividade física $(\mathrm{r}=0,48, \mathrm{p}<0,01)$ e uma relação inversa entre o distúrbio total de humor e o nível de atividade física $(\mathrm{r}=-0,30, \mathrm{p}<0,05)$, da mesma maneira, verificou-se uma relação inversa entre depressão e nível de atividade física, porém não significativa $(\mathrm{p}=0,08)$.

Essas correlações se confirmaram quando comparado os sujeitos com maior nível de atividade física $(n=15)$ com os de menor nível de atividade física $(\mathrm{n}=15)$. Observou-se um maior vigor e um menor distúrbio total de humor naqueles mais ativos do que nos menos ativos. Os fatores negativos do estado de humor tiveram relações positivas entre si e negativas com o vigor, mostrando uma consistência do POMS nessa população.

Tabela 2: Relação entre o Nível de Atividade Física Habitual e as variáveis do Perfil dos Estados de Humor de jovens estudantes do ensino fundamental e médio de uma escola estadual da cidade de Três Rios - RJ.

\begin{tabular}{|c|c|c|c|c|c|c|c|}
\hline & Tensão & Depressão & Raiva & Vigor & Fadiga & $\begin{array}{l}\text { Confusão } \\
\text { Mental }\end{array}$ & $\begin{array}{l}\text { Distúrbio de } \\
\text { Humor }\end{array}$ \\
\hline NAFH & $-0,16$ & $-0,27$ & $-0,06$ & $0,48 * *$ & $-0,23$ & $-0,22$ & $-0,30^{*}$ \\
\hline Tensão & & $0,71 * *$ & $0,81 * *$ & 0,01 & $0,55^{* *}$ & $0,75 * *$ & $0,83 * *$ \\
\hline Depressão & & & $0,67 * *$ & $-0,33^{*}$ & $0,64 * *$ & $0,86^{* *}$ & $0,93 * *$ \\
\hline Raiva & & & & 0,10 & $0,54 * *$ & $0,69 * *$ & $0,80 * *$ \\
\hline Vigor & & & & & $-0,47 * *$ & $-0,15$ & $-0,38^{*}$ \\
\hline Fadiga & & & & & & $0,54 * *$ & $0,79 * *$ \\
\hline Confusão Mental & & & & & & & $0,86^{* *}$ \\
\hline
\end{tabular}

(** Correlação significante para $\mathrm{p}<0,01 ;$ * Correlação significante para $\mathrm{p}<0,05$ )

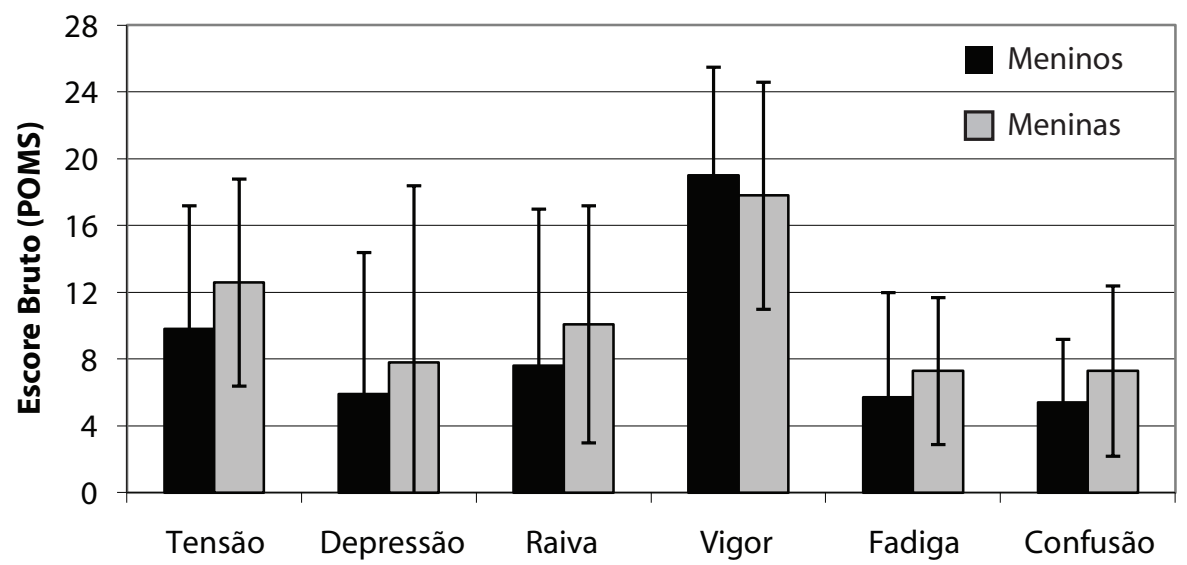

Figura 1. Comparação das variáveis de humor (média e desvio-padrão) em adolescentes estudantes do ensino fundamental e médio de uma escola estadual da cidade de Três Rios - RJ. (Não houve diferença estatisticamente significativa entre as variáveis: $\mathrm{p}>0,05$ ) 


\section{Discussão}

O objetivo do presente estudo foi verificar a relação entre o nível de atividade física relatado por adolescentes e o estado de humor, observando possíveis diferenças entre os gêneros. Observou-se que um maior nível de atividade física em jovens relacionou-se com um maior nível de vigor e menor distúrbio total de humor. Estes dados corroboram a literatura disponível que demonstra que indivíduos ativos, quando comparados aos sedentários ou com indivíduos com um menor nível de atividade física, reportam um melhor estado de humor (Hassmén et al., 2000; Lobstein et al., 1983; Thirlaway \& Benton, 1992). Da mesma forma, houve uma relação inversa entre nível de atividade física e depressão, embora não significativa, que suporta os achados de Sallis, Prochaska e Taylor (2000). Estes estudos de caráter transversal e correlacional mostram que sujeitos que participam de atividades físicas freqüentes reportam menos sintomas de depressão, raiva e estresse percebido, havendo uma associação positiva entre a prática regular de exercícios e o bem-estar psicológico. Os dados do presente estudo demonstram que jovens que relatam maior nível de atividade física relatam também melhor perfil de humor.

Em razão do tipo de estudo transversal e das limitações do método, os resultados encontrados no presente estudo devem ser analisados com cautela, pois não sugerem uma relação de causa e efeito nem de dose-resposta entre o exercício e o estado de humor, mas apenas informa que existe uma associação entre eles. Estudos experimentais randomizados mais recentes nesta área já suportam a relação de causa e efeito, mas não ainda a relação de dose-resposta.

Um dos aspectos positivos do presente estudo é que corrobora as evidências advindas de pesquisas com adultos, na medida em que suporta o potencial benefício da prática regular da atividade física sobre o estado de humor em indivíduos mais jovens. Dessa maneira, os benefícios psicológicos do exercício parecem acontecer independentemente da idade de seus praticantes, corroborando os estudos de Thirlaway e Benton (1992) e Hassmén et al. (2000). Este aspecto indica um fator importante do exercício enquanto promotor do desenvolvimento tanto físico quanto psicológico dos jovens, devendo ser estimulado desde cedo, pois sabe-se que o nível de atividade física diminui cerca de $50 \%$ entre os seis e 16 anos de idade, declinando ainda mais na idade adulta (Sallis et al., 1992). Estes autores afirmam que embora o conhecimento dos benefícios do exercício parecem não ser importantes para a adoção de um estilo de vida mais ativo, o conhecimento de como ser fisicamente ativo pode ser uma importante estratégia de promoção do exercício em jovens.

Da mesma maneira, parece que os benefícios da atividade física são similares tanto para os meninos quanto para as meninas. No presente estudo, não foram encontradas diferenças significativas no perfil de humor na comparação entre gêneros, o que suporta os achados de outros estudos (Hassmén et al., 2000; Newcombe \& Boyle, 1995; Thirlaway \& Benton, 1992). Parece que os efeitos psicológicos do exercício acontecem de forma similar em meninos e meninas assim como acontecem os efeitos fisiológicos.

Como observado em outros estudos, os meninos apresentaram um maior nível de atividade física do que as meninas (Sallis et al., 1992; Sallis et al., 2000). No entanto, essa maior prática de exercício não mostrou influência sobre o estado de humor, sugerindo que os benefícios psicológicos do exer- cício acontecem independentemente do gênero. Destaca-se, porém, que foi observada uma tendência do estado de humor dos meninos ser melhor do que o das meninas, podendo ser uma indicação de que indivíduos com maior freqüência em atividades físicas possuem uma melhor saúde mental do que aqueles com menor freqüência. Por isso, mais estudos são necessários na relação entre a quantidade de prática de exercício e o benefício psicológico.

Em relação à explicação da relação entre exercício e humor, ainda não existe um consenso sobre os mecanismos envolvidos neste fenômeno, porém acredita-se que os benefícios psicológicos do exercício ocorram tanto em função de fatores fisiológicos, como o aumento da aptidão física, aumento do nível de endorfina, serotonina e ondas cerebrais alfa, como por fatores psicológicos e sociais, como o aumento da auto-estima, auto-eficácia, sensação de prazer, contato com as pessoas (Werneck, Bara Filho \& Ribeiro, 2005). Numa sociedade onde há uma tendência cada vez maior à hipocinesia, é importante que a atividade física seja estimulada tanto para seus benefícios fisiológicos como para os psicológicos, procurando alcançar um equilíbrio psicofisiológico harmonioso que contribua no desenvolvimento do adolescente.

Um dos aspectos importantes para a promoção do exercício e para a saúde pública é que a melhoria da aptidão física não parece ser condição primordial para que se obtenha benefícios psicológicos. Dessa forma, intensidades e durações mais elevadas usadas para a melhoria da aptidão física, podem ser substituídas por intensidades e durações menores. Estudos mostram que a participação em atividades físicas mais do que a melhoria da aptidão física está relacionada à melhoria do humor e do bem-estar psicológico (Hassmén \& cols., 2000; Thirlaway \& Benton, 1992). Além disso, Hanssen e cols. (2001) demonstraram que para alcançar os benefícios psicológicos do exercício bastam dez minutos de exercício por sessão. A repetição dessas sessões traria os efeitos crônicos em longo prazo, como foi observado no estudo de Steinberg e cols. (1998). Estes dados são importantes indicadores de prescrição do exercício visando uma melhor saúde mental, em que se recomendam exercícios de moderada intensidade, com sessões não muito longas (10 a 40 minutos) e mais freqüentes durante a semana, devendo ser praticado num ambiente agradável e de forma prazerosa.

Em função do instrumento de medida da atividade física utilizado, os resultados do presente estudo devem ser interpretados com cautela. De acordo com Nahas (1996), existe uma dificuldade na mensuração objetiva e precisa do nível de atividade física habitual, principalmente em grandes populações. Esta dificuldade está nítida num recente estudo de revisão, onde $69 \%$ dos estudos que analisaram a correlação de variáveis e a atividade física de adolescentes utilizaram questionários não-validados, tendo apenas $4 \%$ utilizado medidas objetivas.

Em face dos resultados encontrados no presente estudo e da literatura disponível, pode-se concluir que os participantes que relataram maior nível de atividade física habitual apresentaram melhor escore no estado de humor, independente do gênero. Tanto meninos quanto meninas adolescentes que relatam um maior nível de atividade física habitual relatam também um melhor estado de humor. O estudo também indicou uma possível relação entre a quantidade de exercício praticado e o estado de humor, que deve ser abordada em futuras investigações, explorando relações de causa e efeito. 
A adoção de um estilo de vida mais ativo, através da prática da atividade física regular, tem se mostrado um fator eficaz na prevenção de doenças e na promoção da saúde tanto física quanto mental, devendo, por isso, ser estimulada durante toda a vida. Neste sentido, cabe aos profissionais do exercício e do esporte o dever de fazer com que um número cada vez maior de pessoas adquira o hábito de se exercitar, a começar pela educação física nas escolas, de modo que crianças, jovens e adultos possam desfrutar os benefícios que o exercício proporciona. Diferentes tipos e intensidades de atividade física têm o potencial de melhorar o humor, desde que esteja adequada à individualidade do praticante, seja praticada num ambiente agradável e de forma prazerosa. O benefício psicológico do exercício está na dependência de uma interação ótima entre o praticante, as características do exercício e do ambiente.

\section{Referências}

Brandão, M. R. F., Andrade, D. R., Matarazo, F., Vasques, L., \& Vasconcelos, E. G. (1993). Perfil Psicológico dos Estados de Humor. [Resumo]. II Congresso Interno do Instituto de Psicologia da USP (F1). São Paulo: USP

Dilorenzo, T. M., Bargman, E. P., Stucky-Ropp, R., Brassington, G. S., Frensch, P. A., \& LaFontaine, T. (1999). Long-term effects of aerobic exercise on psychological outcomes. Preventive Medicine, 28, 75-85.

Dunn, A. L., Trived, M. H., \& O’neal, H. A. (2001). Physical activity dose-response effects on outcomes of depression and anxiety. Medicine and Science in Sport and Exercise, 33(6), 587-597.

Hansen, C. J., Stevens, L. C., \& Coast, J. R. (2001). Exercise duration and mood state: how much is enough to feel better? Health Psychology, 20(4), 267-275.

Hassmén, P., Koivula, N., \& Uutela, A. (2000). Physical exercise and psychological well-being: A population study in Finland. Preventive Medicine, 30, 17-25.

Hills, P., \& Argyle, M. (1998). Positive moods derived from leisure and their relationship to happiness and personality. Personality and Individual Differences, 25, 523-535.

Lampinen, P., Heikkinen, R., \& Ruoppila, I. (2000). Changes in intensity of physical exercise as predictors of depressive symptoms among older adults: an eight-year follow-up. Preventive Medicine, 30, 371-380.

Lobstein, D. D., Mosbacher, B. J., \& Ismail, A. H. (1983). Depression as a powerful discriminator between physically active and sedentary middle-aged men. Journal of Psychosomatic Research, 27(1), 69-76.

McAuley, E., \& Rudolph, D. (1995). Physical activity, aging, and psychological well-being. Journal of Aging and Physical Activity, 3, 67-96.

McNair, D. M., Lorr, M., \& Droppleman, L. F. (1971). Manual for the profile of mood states. San Diego, CA: Educational and Industrial Testing Services.

Nahas, M. V. (1996). Revisão de métodos para determinação dos níveis de atividade física habitual em diversos grupos populacionais. Revista Brasileira de Atividade Física e Saúde, 1(4), 27-37.

Nahas, M. V. (2001). Atividade física, saúde e qualidade de vida: Conceitos e sugestões para um estilo de vida ativo. Londrina: Midiograf.
Newcombe, P. A., \& Boyle, G. J. (1995). High school student's sports personalities: variations across participation level, gender, type of sport, and success. International Journal of Sport Psychology, 26, 277-294.

Petruzzello, S. J., Landers, D. M., Hatfield, B. D., Kubitz, K. A., \& Salazar, W. (1991). A meta-analysis on the anxietyreducing effects of acute and chronic exercise: outcomes and mechanisms. Sports Medicine, 11(3), 143-182.

Sallis, J. F., Simons-Morton, B. G., Stone, E. J., et al. (1992). Determinants of physical activity and interventions in youth. Medicine and Science in Sports and Exercise, 24(6), 248-257.

Sallis, J. F., Prochaska, J. J., \& Taylor, W. C. (2000). A review of correlates of physical activity of children and adolescents. Medicine and Science in Sports and Exercise, 32(5), 963-975.

Slaughter, M. H., Lohman, T. G., Boileau, R. A., Horswill, C. A., Stillman, R. J., Van Loan, M. D., \& Bemben, D. A. (1988). Skinfold equations for estimation of body fatness in children and youth. Human Biology, 60, 709-723.

Steinberg, H., Nicholls, B. R., Sikes, E. A., LeBoutillier, N., Ramlakhan, N., Moss, T. P., \& Dewey, A. (1998). Weekly exercise consistently reinstates positive mood. European Psychologist, 3(4), 271-280.

Stephens, T. (1988). Physical activity and mental health in the United States and Canada: evidence from four population surveys. Preventive Medicine, 17, 35-47.

Strawbridge, W. J., Deleger, S., Roberts, R. E., \& Kaplan, G. A. (2002). Physical activity reduces the risk of subsequent depression for older adults. American Journal of Epidemiology, 156, 328-334.

Terry, P. C., \& Lane, A. M. (2000). Normative values for the profile of mood states for use with athletic samples. Journal of Applied Sport Psychology, 12, 93-109.

Terry, P. C., Lane, A. M., Lane, H. J., \& Keohane, L. (1999). Development and validation of a mood measure for adolescents. Journal of Sports Sciences, 17, 861-872.

Thayer, R. E., Newman, R. \& McClain, T. M. (1994). Self-regulation of mood: strategies for changing a bad mood, raising energy, and reducing tension. Journal of Personality and Social Psychology, 67(5), 910-925.

Thirlaway, K., \& Benton, D. (1992). Participation in physical activity and cardiovascular fitness have different effects on mental health and mood. Journal of Psychosomatic Research, 36(7), 675-685.

U.S. Department of Health and Human Services (1996). Physical activity and health: A report of the Surgeon General. Atlanta: Centers for Disease and Prevention, National Center for Chronic Disease Prevention and Health Promotion.

Werneck, F. Z. (2003). Efeitos psicofisiológicos agudos do exercício aeróbio e contra-resistência em diferentes intensidades. Dissertação de Mestrado, Universidade Gama Filho, Rio de Janeiro.

Werneck, F. Z., Bara Filho, M. G., \& Ribeiro, L. C. S. (2005). Mecanismos de melhoria do humor após o exercício: revisitando a hipótese das endorfinas. Revista Brasileira de Ciência e Movimento, 13(2), 135-144.

Recebido em 05.08.2009

Primeira decisão editorial em 19.07.2010

Versão final em 26.07.2010

Aceito em 06.08.2010 